\title{
Tooth Development Disorder, CTCAE
}

National Cancer Institute

\section{Source}

National Cancer Institute. Tooth Development Disorder, CT CAE. NCI Thesaurus. Code C143876.

A disorder characterized by a pathological process of the teeth occurring during tooth development. 